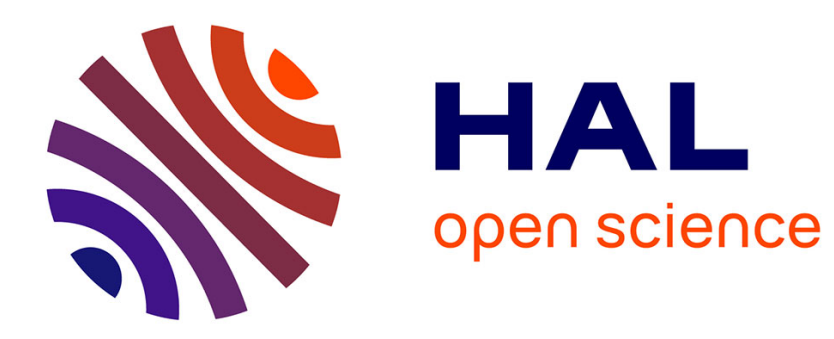

\title{
Inégalités sociales de santé et déni d'agenda. Portraits croisés de trois intellectuels spécifiques
}

\author{
Frédéric Pierru
}

\section{To cite this version:}

Frédéric Pierru. Inégalités sociales de santé et déni d'agenda. Portraits croisés de trois intellectuels spécifiques. Revue française des affaires sociales, 2021. halshs-03507300

\section{HAL Id: halshs-03507300 \\ https://shs.hal.science/halshs-03507300}

Submitted on 3 Jan 2022

HAL is a multi-disciplinary open access archive for the deposit and dissemination of scientific research documents, whether they are published or not. The documents may come from teaching and research institutions in France or abroad, or from public or private research centers.
L'archive ouverte pluridisciplinaire HAL, est destinée au dépôt et à la diffusion de documents scientifiques de niveau recherche, publiés ou non, émanant des établissements d'enseignement et de recherche français ou étrangers, des laboratoires publics ou privés. 


\section{[POINT DE VUE] Inégalités sociales de santé et déni d'agenda. Portraits croisés de trois intellectuels spécifiques [à paraître dans le futur numéro de la RFAS] \\ Frédéric Pierru}

Le 26 septembre 2020, Richard Horton, éditeur en chef du prestigieux Lancet, revue elle-même prise dans la tourmente des publications scientifiques relatives à la Covid-19, publiait un éditorial très politique qui a eu un grand écho dans le monde. Il y remet en cause l'usage de la notion de pandémie pour qualifier la crise sanitaire actuelle, au profit de celle de « syndémie » :

« Deux catégories de maladies s'entremêlent dans certaines populations - l'infection par le SARS-Cov-2 avec des défaillances respiratoires sévères et un éventail de maladies non transmissibles. Ces situations se concentrent dans des groupes sociaux selon des schémas inégalitaires profondément ancrés dans nos sociétés. L'accumulation de ces maladies sur fond d'inégalités économiques et sociales amplifie les effets de chaque maladie. La Covid-19 n'est pas une pandémie. C'est une syndémie. [...] Limiter les dégâts du SARS-Cov-2 demandera davantage d'attention aux maladies non transmissibles et aux inégalités socioéconomiques que cela n'a été le cas jusqu'alors. [...] Les syndémies se caractérisent par l'intrication du social et du biologique, entre des situations [sociales] et des états [de santé], ces interactions augmentant la disposition d'une personne à pénaliser ou à aggraver son état de santé » (Horton, 2020) ${ }^{1}$.

La notion de pandémie suggère que le virus serait égalitaire dans le choix de ses victimes. Or, les études épidémiologiques ont montré que ce n'est pas le cas. Le Sars-Cov-2 dégrade préférentiellement la santé des personnes atteintes de maladies chroniques, lesquelles ne se distribuent pas au hasard dans l'espace social. Il amplifie les inégalités sociales de santé (ISS), elles-mêmes en partie corrélées aux inégalités socio-économiques. À l'évidence, il s'agit là d'un éditorial très politique, dans le contexte post-référendum du Brexit. On en veut pour preuve qu'il fait écho au nouveau rapport Marmot sur « les inégalités de santé en Angleterre » qui dresse un constat sans appel de l'évolution sanitaire du pays :

«La dernière décennie a été marquée par la détérioration de la santé et l'accroissement des inégalités de santé. Les personnes vivant dans les régions les plus pauvres hors de Londres ont vu leur espérance de vie stagner, voire décliner, pendant qu'elle augmentait dans les régions plus avantagées. En ce qui concerne l'espérance de vie en bonne santé, il y a eu une légère augmentation pour les hommes et une diminution légère pour les femmes » (Institute of Health Equity, 2020).

Comment ne pas voir l'étrange concordance entre la géographie électorale du référendum sur le Brexit - les régions les plus défavorisées ayant voté pour la sortie de l'Union européenne pendant que les zones les plus prospères votaient pour le Remain - et l'aggravation des inégalités sociales de santé qui est elle-même un indicateur du déclin économique et social de pans entiers du territoire britannique. Cette préoccupation n'est d'ailleurs pas qu'anglaise. Récemment, dans le contexte du "trumpisme», deux chercheurs de renom, Anne Case et Angus Deaton, ont publié un ouvrage qui, lui aussi, a connu un fort écho, intitulé de façon édifiante, Morts de désespoir avec un sous-titre non moins édifiant: l'avenir du capitalisme (Case et Deaton, 2021). Ils y insistent sur la mortalité entrainée par l'épidémie d'opiacées, les suicides et les cirrhoses alcooliques, particulièrement dans la classe ouvrière blanche, celle-là même qui constitue la base du « trumpisme ». Les deux chercheurs expliquent cette recrudescence de la mortalité par le creusement des inégalités socio-économiques, la

\footnotetext{
${ }^{1}$ Traduction de l'auteur. En raison de la place limitée, nous avons réduit les références bibliographiques au strict minimum.
} 
stratification éducative, les effets délétères de la mondialisation sur les emplois industriels et, enfin, un système de santé dont on sait l'exceptionnalité en termes de coûts et d'inégalités. La santé est bien un fait social total en tant qu'elle met « en branle la totalité de la société et de ses institutions », pour reprendre la notion célèbre de Marcel Mauss.

En résumé, l'aggravation des inégalités sociales et territoriales de santé, en particulier face à la « syndémie » de Covid-19, joue le rôle de révélateur d'inquiétudes montantes sur le creusement des inégalités dans de nombreux domaines (économique, emploi, éducation, logement, etc.) qui, à leur tour, suscitent des traductions politiques inquiétantes en termes de "populisme ». C'est ainsi qu'il faut, selon nous, interpréter l'accumulation de prises de position comme celles de Horton, Marmot, Case et Deaton ou encore de Pickett et Wilkinson (2013).

En France, ces prises de position ont connu un certain écho : des chercheurs ou encore des intellectuels se sont emparés de la notion de « syndémie » pour en faire des tribunes, des articles et des «tracts » (Stiegler, 2020), tous soulignant que la mort par Covid-19 ne frappe pas au hasard: elle suit les lignes des «fractures françaises », même si la France ne connaît pas (encore ?) les niveaux inégalitaires du monde anglo-saxon. Dans l'Hexagone, ces prises de position soulignent l'ampleur des inégalités face aux maladies chroniques qui constituent un terreau favorable pour les formes sévères de la Covid-19. Car, faut-il le rappeler, la France est le pays où les inégalités de mortalité et de santé sont les plus élevées en Europe occidentale, et celles-ci, après une période de stagnation, recommencent à se creuser. Les travaux des géographes de la santé, comme E. Vigneron, sont, sur ce point, sans appel : ces inégalités sociales et territoriales de santé et d'accès aux soins sont historiques et durables. Dès 1994, le Haut Conseil de la santé publique tirait la sonnette d'alarme : «la croissance des écarts en termes d'indicateurs de santé est une caractéristique péjorative et alarmante de l'état de la santé en France. Les disparités de la consommation de soins se sont pourtant réduites au cours des trente dernières années » (Haut conseil de la santé publique, 1994).

\section{De la permanence du déni d'agenda en France}

L'on pourrait se réjouir de cette "prise de conscience » à la faveur de la pandémie. Mais il semble que l'inscription de la réduction des inégalités sociales de santé à l'agenda politique, à la faveur de la syndémie de Covid-19, ne soit pas acquise, loin de-là. On en veut pour preuve la surprenante étude publiée le 6 juillet 2021 par l'Institut national du cancer, Santé publique France, le Réseau des registres des cancers et le service biostatistique des hospices civils de Lyon (Santi, 2021). Surprenante car y sont mises en avant les inégalités de genre, qui restent fortes, mais quasiment pas les inégalités sociales ! Or, toutes les études montrent que le statut socio-économique constitue un facteur pronostic important pour la plupart des cancers. Et à pathologie identique, quelle que soit la localisation, le pronostic est toujours moins bon pour les groupes sociaux défavorisés. Plus généralement, les inégalités sociales face au cancer, de l'incidence au traitement, demeurent considérables (Loretti, 2021). Les choix ayant présidé à une telle étude interrogent le politiste : alors que les pays anglo-saxons, réputés plus libéraux et inégalitaires, amorcent une critique acerbe des conséquences sanitaires et politiques des inégalités socio-économiques, la France, avec son État fort et sa "passion pour l'égalité », demeure globalement indifférente aux inégalités sociales de santé.

Comme l'a souligné le chercheur en épidémiologie sociale Thierry Lang, cette cécité ou ce «fatalisme» français face aux ISS sont structurels en France (Haut Conseil de la santé publique, 2009 ; Lang, 2015). Malgré des prises de position politiques fortes à partir de 2009 en matière de réduction des ISS, celles-ci sont restées largement incantatoires et se sont 
résumées à des politiques d'accès à la prévention et aux soins pour les « publics précaires ». Un rapport européen coordonné par Michael Marmot, publié en 2013, situait la France dans le second groupe (sur trois) en matière de politiques de réduction des ISS (Lang, 2015). Même dans le domaine de la recherche, très dynamique sur ce sujet, les chercheurs travaillant sur les ISS constituent un groupe restreint et relativement marginal, Thierry Lang les qualifiant, avec une part d'autodérision, d' « inéquologistes » (Lang, 2018). Là où dans de nombreux pays l'étude des ISS et des moyens de les réduire constitue l'épine dorsale de la santé publique, elle demeure en France un sujet parmi d'autres pour les spécialistes en la matière.

Certes, des progrès considérables en termes de connaissance ont été accomplis depuis les recherches pionnières de Gérard Calot, Guy Desplanques ou de Pierre Aïach, en France. Les avancées de l'épigénétique et de la life course epidemiology ont été, depuis vingt ans, décisives. Les chercheurs français se sont inscrits dans des réseaux internationaux très dynamiques dans lesquels la notion d'embodiment (incorporation), la socialisation du biologique et la biologisation du social - notion qui ne peut que rencontrer l'intérêt d'un sociologue formé à l'école de Pierre Bourdieu et de Luc Boltanski et, aujourd'hui, par exemple, de Bernard Lahire, est devenue cardinale : la santé se construit tout au long de la vie, les premières années étant déterminantes, et dans des cercles concentriques qui vont des gènes et du recours aux soins à l'environnement social plus global (conditions de travail - ou de non travail, allant de l'éducation, en passant par le logement, la qualité du «lien social » - Durkheim aurait parlé d'intégration sociale) jusqu'à, bien entendu, l'exposition aux substances chimiques et à la pollution. Réduire le gradient social de santé implique des actions à tous les niveaux, mais l'on peut résumer ainsi les leçons pour l'action publique de cet ensemble impressionnant de travaux : les ISS ne peuvent être réduites dans des sociétés de plus en plus inégalitaires, et pas seulement sur le plan socio-économique. Les travaux de l'Observatoire des inégalités sont, de ce point de vue, sans appel en ce qui concerne le cas de la France : elles se creusent dans de nombreux secteurs de l'action publique (Maurin, 2021). Pendant ce temps, les géographes, pas seulement de la santé, interrogent la dynamique de « métropolisation » qui laisse sur le bas-côté ce qu'il est désormais convenu d'appeler abusivement la " France périphérique », des zones péri-urbaines aux zones rurales. Emmanuel Vigneron a ainsi montré comment les indicateurs de santé variaient selon un schéma centre-périphérie, prenant l'exemple frappant de leur évolution le long de la ligne D du RER parisien.

Dès lors, il semble difficile de parler « d'agnotologie » en matière d'ISS, même si le rapport précité sur les inégalités face au cancer semble y succomber, et cela-même après les « leçons » de la syndémie de Covid-19. Les connaissances sont-là, elles appellent certes de plus amples développements et affinements, mais elles sont suffisamment robustes pour en tirer des conclusions de politique publique. La question reste donc pleine et entière pour le chercheur en sciences sociales : pourquoi une telle faiblesse de l'action publique dans un domaine, la santé, alors que nous vivons dans une certaine "idolâtrie de la vie », selon le mathématicien et philosophe Olivier Rey? (Rey, 2020). Une idolâtrie qui peut conduire à confiner des millions d'individus afin de protéger les personnes les plus âgées et celles dites « fragiles », c'est-à-dire celles qui ont des états de santé dégradés, notamment par des maladies chroniques, et dont certaines furent pourtant les «premiers de corvée »? L'organisation de la « résilience »- la preparedness disent les spécialistes - d'une société ne se résume pas à la seule économie. Elle est aussi sanitaire : lutter contre l'actuelle épidémie de maladies chroniques ce n'est pas seulement éviter un lourd tribut pour les finances sociales, c'est aussi permettre à la société d'être moins vulnérable face aux «nouvelles » menaces infectieuses, dont tous les spécialistes disent que le Sars-Cov-2 n'est que le prélude, au regard des avancées de l'urbanisation, de la déforestation et du réchauffement climatique notamment. À l'heure où nous écrivons ces lignes, 
au début de l'été 2021, le gouvernement s'inquiète de la diffusion du variant « indien » ou delta du Covid-19, tandis que de nombreux pays prennent de nouvelles mesures restrictives. L'été 2021 ne sera pas aussi tranquille sur le front pandémique que l'été 2020.

Ce grand écart entre les connaissances et l'action publique doit donc être interrogé en ce qu'il constitue, ce que l'on appelle en science politique, un phénomène de « déni d'agenda » et de " non décision » (Cobb et Ross, 1997). De prime abord, les facteurs vont du niveau le plus global aux niveaux les plus sectoriels, sanitaire et médical.

\section{Le mort saisit le vif}

Les politistes de l'école "grenobloise », qualifiée d'approche cognitive des politiques publiques, ont avancé l'idée que les politiques publiques sectorielles étaient structurées par des « référentiels »-des conceptions contingentes des problèmes et des façons de les résoudre -, eux-mêmes encapsulés dans des institutions et des instruments, défendus par des groupes d'intérêt et dominés par un «référentiel global» qui donne un «air de famille » à l'action publique quel que soit le secteur concerné. Cette école a ainsi documenté la réfraction sectorielle du « tournant néolibéral » (Jobert, 1994), qualifié de gestionnaire dans sa version française, dans les années 1980. Dans un contexte de libéralisation des biens et des capitaux, mais aussi d'un chômage structurel de $10 \%$, la compétitivité et l'attractivité deviennent les maître-mots de l'action publique, ce qui suppose notamment d' " assouplir » le droit du travail et de maîtriser les dépenses publiques. Les inégalités sociales changent de sens : au lieu d'être des anomalies qu'il convient de combattre par des politiques publiques volontaristes, comme elles l'étaient dans le paradigme keynésiano-fordiste, elles sont désormais perçues comme des facteurs de dynamisme économique. L' « équité », l' " égalité des chances » (Maurin, 2021 ; Sandel, 2021) ou encore l'« exclusion» (Castel, 1995) remplacent «l'exploitation » de la « lutte des classes » dans les catégories dominantes du débat public.

Ces transformations globales percutent les institutions du système de santé qui font montre pourtant d'une inertie certaine. D'un côté, elles en renforcent quelques caractéristiques historiques. En effet, le sous-espace de la «médecine sociale » est très largement dominé par celui de la clinique, au moins depuis le début du $\mathrm{XX}^{\mathrm{e}}$ siècle, malgré les succès du mouvement hygiéniste au XIX ${ }^{\mathrm{e}}$ siècle (Pinell, 2005). L'approche populationnelle de la santé est depuis longtemps marginalisée au profit d'une vision des biens de santé s'échangeant entre une demande individuelle - le patient - et une offre professionnelle, la relation thérapeutique étant solvabilisée par les fonds parapublics de la Sécurité sociale. L'existence d'une offre de soins largement privée - médecine libérale, établissements privés à but non lucratif, cliniques privées commerciales - est en phase avec la célébration du « privé » contre un secteur public désormais dépeint comme trop bureaucratique, lourd et, surtout, coûteux. De plus, la biomédecine des centres hospitalo-universitaires est désormais célébrée, non seulement pour sa promesse de maîtrise des coûts - en fait, le «progrès médical » s'avère être le premier facteur d'augmentation des dépenses de santé - mais aussi pour son potentiel économique et industriel en termes d'innovations. Le réductionnisme biomédical marginalise encore plus l'approche globale et populationnelle de la santé. L'impératif de la maîtrise des dépenses publiques de santé, qui s'est particulièrement affirmé au cours de la dernière décennie, se révèle finalement très conservateur: loin de contribuer au changement, ou du moins à la relativisation, du paradigme médico-centré dominant, il en renforce au contraire les principaux traits. Ces évolutions atteignent aussi le sous-espace de la médecine sociale, c'est-à-dire de la santé publique. 
Des savoirs de gouvernement nouveaux émergent, sont importés et s'imposent, les uns visant à modifier les comportements individuels (le comportementalisme du fameux nudging [Bergeron et al., 2018] ou encore l'épidémiologie des facteurs de risque), les autres à permettre à l'État d'assurer ses missions régaliennes de sécurité, comme l'épidémiologie de veille sanitaire. Dans une telle configuration, l'épidémiologie dite "sociale», portant sur les déterminants sociaux de la santé, ou la conception de la santé publique comme disciplinecarrefour entre épidémiologie et sciences sociales sont plus marginalisées que jamais. Les sciences sociales sont particulièrement démonétisées dans le cadre d'une vision dominante de la société considérée comme un agrégat d'individus rationnels et responsables surplombé par un Léviathan sécuritaire.

D'un autre côté, cependant, les transformations des catégories dominantes de l'action publique se réfractent dans les politiques de santé. Il en est ainsi du schème de l'exclusion. La vision dichotomique de la société entre exclus et inclus conduit à considérer la lutte contre les ISS comme une politique d'accès à la prévention secondaire et aux soins des publics précaires, c'est-à-dire les plus éloignés des filières classiques. C'est ainsi qu'au sein des agences régionales de santé (ARS), des programmes régionaux pour l'accès à la prévention et aux soins des plus démunis sont mis en œuvre selon ce principe. Il ne s'agit pas de nier l'intérêt de ces dispositifs - qui demeurent néanmoins périphériques au sein de l'administration sanitaire, mais de souligner que la problématique du gradient social de santé n'y trouve guère son compte. La lutte contre les ISS ne saurait se résumer à l'accès à la prévention et aux soins des « exclus ", comme le montre l'évolution des indicateurs de santé. C'est la pertinence du principe du « ciblage » des politiques sanitaires et sociales sur " ceux qui en ont le plus besoin », qui fait consensus à gauche et à droite depuis les années 1990 (Palier, 2005), qui doit être interrogée.

\section{Trois engagements de chercheurs scandalisés par «l'inégalité des vies » (Fassin, 2020)}

Dans ce contexte institutionnel et politique défavorable, des chercheurs tentent d'ouvrir une brèche dans les rapports de force et de sens qui structurent les politiques publiques de santé afin d'y imposer une conception alternative de l'action publique, moins médicocentrée, plus populationnelle et, surtout, attentive au gradient social de santé. Bref, ils essaient de forcer le " déni d'agenda », mais selon des postures et des répertoires d'action différents. Nous en avons retenu trois, avec une part d'arbitraire, mais pas seulement, car ils comptent parmi les plus en vue, avec lesquels nous nous sommes entretenus pour cet article. Leur point commun est évidemment d'adhérer à une conception populationnelle de la santé avec une sensibilité sociale marquée. Pourtant, leur engagement en tant qu' « intellectuel spécifique », au sens de Foucault, notion revisitée par Gérard Noiriel ${ }^{2}$, n'est pas identique. Il va du plus savant (Thierry Lang) au plus «critique » (Annie Thébaud-Mony), en passant par une posture intermédiaire, celle du chimiste et toxicologue André Cicolella.

Thierry Lang, issu d'un milieu modeste, en a gardé une « fibre sociale ». Devenu médecin, il lit un travail montrant qu'à l'hôpital les ouvriers sont reçus par les internes, là où les cadres bénéficient d'une consultation avec les chefs de service, ce qu'avait montré la sociologue Antoinette Chauvenet dans les années 1970. Cette rencontre entre des dispositions, une époque (les années contestataires 1960-1970) et cette lecture le conduit à s'orienter vers l'étude des

\footnotetext{
${ }^{2}$ «L'intellectuel spécifique est un universitaire qui accepte de prendre la parole en public pour poser autrement les problèmes d'actualité car il est convaincu que, dans une société démocratique, l'enjeu décisif des luttes de pouvoir se situe du côté des questions plutôt que des réponses. » (Noiriel, 2010). Les questions d'actualité sont ici les questions sanitaires. Durkheim, Bloch, Foucault ou encore Bourdieu en sont, avec des variations, des représentants éminents.
} 
inégalités de santé. Son investissement dans l'épidémiologie - et peut-être le peu de goût pour la clinique, mais il s'agit là de notre interprétation - sera marqué de façon indélébile par la sensibilité à la question sociale. Pour Thierry Lang, la lutte contre les ISS passe d'abord, et avant toute chose, par l'accumulation de connaissances robustes et scientifiquement validées. $\mathrm{Au}$ fond, chez lui, il y a un optimisme de la connaissance, en dépit de la sentence de Spinoza selon laquelle «il n'existe pas de force intrinsèque des idées vraies », phrase que Pierre Bourdieu estimait être la plus triste de la philosophie. De fait, Thierry Lang, devenu professeur de médecine à Toulouse, s'emploie non seulement à lancer des recherches selon les canons de la scientificité biomédicale, mais il s'inscrit aussi dans des réseaux internationaux qui favorisent l'importation en France des résultats produits dans des pays plus sensibles à la problématique du gradient social de santé. Pour autant, sa posture de savant en pointe en matière de connaissances de life course epidemiology et d'épigénétique ne le cantonne pas à se satisfaire de la tour d'ivoire académique. En 2000, il codirige la publication, à La Découverte, d'un volumineux ouvrage qui fait la synthèse des connaissances disponibles sur les ISS (Leclerc et al., 2000). Cet ouvrage est suivi de la publication, chez le même éditeur, d'un autre livre, plus " grand public » en 2008 (Leclerc et al., 2008). La même année, il saisit l'opportunité de créer un groupe " inégalités sociales de santé » au sein du Haut Conseil de santé publique, soit un lieu « neutre » ou d'interface entre l'espace scientifique et l'espace de la décision publique, bref le monde de l'expertise. C'est à ce titre qu'il rédige un rapport retentissant au titre éloquent : Inégalités sociales de santé : sortir de la fatalité (Haut Conseil de la santé publique, 2009).

Au même moment, une nouvelle réforme des retraites étant envisagée, le constat d'inégalités d'espérance de vie en bonne santé qui suivent la hiérarchie sociale est « naturalisé »: loin de susciter une forme d'indignation, elles sont considérées comme des paramètres dont il faut tenir compte pour le calcul des pensions. Thierry Lang oppose aux choix publics la conclusion des résultats internationaux en matière d'ISS : la polarisation sur l'accès aux soins, y compris sur le mode compassionnel des " exclus », est incapable de réduire le gradient social de santé, surtout au moment où la réforme Hôpital patient santé territoire (HPST) autonomise la santé du social, avec la création des ARS puisque les déterminants sociaux de la santé sont à chercher ailleurs que dans le système de soins. Une telle autonomisation du secteur de la santé, dont les mobiles liés à la maîtrise de la trajectoire des finances publiques sont extérieurs au secteur, conduit à redéfinir profondément la santé publique. Cette dernière se restreint alors aux incitations à la modification des comportements individuels, moyennant l'importation des nudgings, ou à l'espérance d'agir, sur un mode thérapeutique biomédical, sur les conséquences de l'environnement sur les gènes alors que l'épigénétique a démontré l'interaction complexe entre gènes et environnement. De fait, les institutions du système de santé français peuvent s'accommoder de tels résultats, pourtant dérangeants, pour en déduire des politiques publiques compatibles avec le legs historique. Pourtant, les avancées récentes en matière de connaissances relatives à la construction sociale de la santé tout au long de la vie, et singulièrement aux premiers âges (Lang et al., 2020), posent des questions éthiques considérables. Outre celle, classique, entre responsabilités individuelle et collective, elles questionnent la solidarité entre générations : d'un côté la dette publique conduit, au nom des générations futures, à sacrifier les politiques sociales, lesquelles pourtant participent de la réduction des ISS, mais, de l'autre, il faudrait restreindre les libertés (de fumer ou boire, par exemple) des générations actuelles au nom, toujours, de l'état de santé des générations les plus récentes. La contradiction saute aux yeux. On ne peut s'empêcher de penser à la fameuse phrase de Marx : «L'Humanité ne se pose jamais que les problèmes qu'elle peut résoudre. »

À l'autre extrémité du spectre, à la limite de l'intellectuelle spécifique et critique, la sociologue Annie Thébaud-Mony a consacré une grande partie de sa carrière à étudier et à dénoncer les 
inégalités d'exposition aux risques professionnels ${ }^{3}$. Formée à la sociologie, à l'école de Bourdieu-Passeron des Héritiers - «pour moi la sociologie, ce sont les inégalités » dit-elle -, elle s'est d'abord intéressée aux inégalités scolaires dans différents pays, son conjoint d'alors étant diplomate : Brésil, Sénégal, Algérie. C'est au début des années 1980, dans le cadre d'échanges franco-brésiliens, qu'elle commence à investir le thème de l'emploi, de la division $\mathrm{du}$ travail et des risques, en prenant contact avec le collectif « risques et maladies professionnelles » animé par celui qui allait devenir son compagnon, Henri Pézerat, et les militants anti-amiante du collectif intersyndical des universités Jussieu. Sa première enquête porte sur les accidentés du travail dans les centres de distribution d'EDF, où elle découvre la division du travail et des risques entre donneurs d'ordre et sous-traitants. Pour la sociologue, à la différence du médecin Thierry Lang, il y a une continuité entre le travail d'enquête sociologique et l'engagement aux côtés des syndicats et des collectifs citoyens qui dénoncent des conditions de travail particulièrement nocives pour la santé. Connaissances sociologiques et demandes syndicales se nourrissent les unes les autres et le lien entre recherche, action et engagement se fait «naturellement»: la sociologue ne peut rester distanciée face aux récits biographiques des nombreux individus ayant vécu un accident du travail ou contracté une maladie professionnelle. Qu'il s'agisse d'EDF, des sites miniers ou encore de l'amiante, Annie Thébaud-Mony s'indigne de l'invisibilisation non seulement des risques professionnels mais, plus précisément encore, des profondes inégalités face à ces derniers, le monde du travail précaire, intérimaire notamment, et de la sous-traitance, avec ses nombreux travailleurs immigrés, subissant de plein fouet l'exposition aux substances toxiques. En quelque sorte, l'invisibilité des inégalités face aux risques professionnels trouve sa cause principale dans la division du travail de production et de reproduction. De surcroît, l'existence d'un chômage structurel de masse conduit les décideurs publics à privilégier l'emploi à tout prix, aux dépens du travail et des conditions concrètes de son effectuation. Si certains syndicats locaux se saisissent de la question des risques professionnels, de même que des collectifs citoyens, par exemple contre les pesticides, les grandes confédérations syndicales ont tendance, elles aussi, à privilégier la sauvegarde de l'emploi et, de moins en moins, la défense du pouvoir d'achat.

Enfin, dans le champ-même de la connaissance, et ici Annie Thébaud-Mony rejoint le constat de Thierry Lang, le thème des inégalités sociales de santé n'est guère valorisé dans des grandes institutions, comme l'Institut national de la santé et de la recherche médicale et l'Institut national du cancer, au sein desquelles la place des sciences sociales est de plus en plus discutée. La généralisation des appels à projet n'a fait que renforcer ce point aveugle de la connaissance et consacrer les disciplines déjà les plus renommées (épidémiologie des facteurs de risque ou encore l'Evidence based medicine). Dans le cadre de ce cas d'école «d'agnotologie » et d'inaction publique (Henry, 2017), la sociologue qui s'intéresse et s'indigne face à la dégradation des conditions de travail doit être pleinement en phase avec les luttes locales (France Télécom, AZF, collectif Lubrisol) et aider les victimes à être reconnues, tant par la Sécurité sociale que, parfois, la justice pénale. Ses actions peuvent aller avec des coups d'éclat, comme le refus, en 2012, de la légion d'honneur que voulait lui décerner la ministre de l'Égalité, des Territoires et du Logement, Cécile Duflot. Elles mènent surtout la sociologue à une critique du capitalisme globalisé qui la rapproche de la figure de l'intellectuelle révolutionnaire identifiée par Gérard Noiriel.

Le cas du chimiste et toxicologue André Cicolella est intermédiaire entre l'intellectuel spécifique et l'intellectuel critique. Plus précisément, après avoir été licencié abusivement par l'Institut national de recherche et de sécurité pour avoir lancé un programme de recherche sur

\footnotetext{
${ }^{3}$ Son itinéraire est retracé dans Thébaud-Mony, 2021.
} 
les éthers de glycol, il a contribué à faire le portrait de l'intellectuel spécifique en « lanceur d'alerte », notion qu'il va contribuer à imposer, avec d'autres, dans le droit français. Il n'est, à n'en pas douter, aussi conscient que Thierry Lang et Annie Thébaud-Mony du gradient social de santé et de ses déterminants sociaux. Comme ces derniers, et c'est en cela qu'il est aussi un intellectuel spécifique, il s'appuie sur les avancées scientifiques et refuse la posture de la simple dénonciation politique non adossée à des connaissances robustes et validées :

«Tu dénonces, ok, mais après qu'est-ce que tu fais, si tu es tout seul ? Rien. Moi, ça ne m'intéresse pas. Mon objectif c'est d'obtenir une meilleure protection de la santé humaine parce que c'est l'intérêt bien compris de tout le monde, en dépit des clivages politiques qui ont leur légitimité par ailleurs. Moi, je ne suis pas gêné d'aller signer avec Valérie Pécresse une action de sensibilisation aux perturbateurs endocriniens dans les lycées, alors que j'ai eu des responsabilités chez les Verts. »

Comme eux, il s'efforce de déstabiliser un système de santé médicocentré et biomédical qui ne conçoit les patients que comme désencastrés de leur environnement. Il faut selon lui passer d'une politique de soins à une politique de santé, et d'une assurance-maladie à une assurancesanté. De la même façon, il critique l'approche tronquée des institutions publiques comme la Caisse nationale d'assurance maladie, dans son rapport produits et charges par exemple, ou encore la Cour des comptes, qui voient dans la prévalence croissante des maladies chroniques un effet mécanique du vieillissement démographique et des avancées biomédicales (la chronicisation du cancer, par exemple).

Pour autant, son engagement public en faveur de la «santé environnementale », notion structurante dans sa réflexion, suit une autre stratégie. En effet, ayant tiré des conclusions de l'affaire de l'amiante, il ne met délibérément pas en avant, par souci d'efficacité politique, le thème des inégalités pour mieux insister sur le fait que tout le monde est exposé à des substances chimiques toxiques (Cicolella, 2013). Tout un chacun doit donc se sentir concerné personnellement par la pollution chimique environnementale. À l'instar de Thierry Lang, il insiste, avec la notion d'exposome, sur le fait qu'une partie importante des maladies trouvent leur origine dans une exposition aux stress environnementaux pendant la période de gestation et de l'enfance. Avec un tel cadrage, il s'agit de donner une audience maximale aux études sur les effets sanitaires délétères de la prolifération des substances chimiques toxiques. Il rappelle à cet égard que la chimie est une science avant d'être une industrie et que cette science peut être très bien mise au service de la recherche de substances propres. À ce titre, il a participé à l'interdiction de l'usage du perchloéthylène dans les pressings au profit d'un solvant propre : l'eau. De plus, son approche est résolument pragmatique, d'une façon parfois différente de celles de Lang ${ }^{4}$ et Thébaud-Mony. Il a créé le Réseau environnement santé (RES) qui a élaboré une charte énonçant des principes fondamentaux et qui noue des partenariats avec des acteurs locaux, notamment des collectivités territoriales. Dernière action en date : une campagne de sensibilisation aux perturbateurs endocriniens dans dix lycées d'Île-de-France, avec le soutien du conseil régional : les lycéens étaient incités à porter un bracelet en silicone de façon à mettre en évidence qu'ils étaient exposés à des substances comme les phtalates. En dernier lieu, sa stratégie consiste à mettre en avant le coût économique exorbitant de l'épidémie de maladies chroniques, lui-même dénoncé par l'Organisation mondiale de la santé depuis 2011 : en 2014, 65 milliards d'euros en plus ont été dépensés, en France, pour les maladies chroniques par rapport à 1994. Dès lors, une action résolue sur les facteurs environnementaux des maladies chroniques afin d'en réduire l'incidence permettrait de réaliser de substantielles économies.

En conclusion, nos trois chercheurs engagés partagent, par-delà tout ce qui les sépare (formation, trajectoire sociale et professionnelle), un socle commun de convictions: la

\footnotetext{
${ }^{4}$ Précisons que Thierry Lang et son équipe ont eux aussi mené des recherches-actions avec des acteurs institutionnels ou non de la région toulousaine.
} 
dénonciation ne suffit pas, il faut adosser les prises de position publiques aux avancées de la connaissance scientifique en matière d'interactions entre santé et environnement - un champ de recherche dynamique qui, pourtant, ne connaît que très peu de traductions du point de vue de l'action publique, singulièrement en France. De surcroît, ce décalage constitue pour les trois chercheurs un scandale qui suppose un engagement public prenant des formes différentes : publication de tribunes, ouvrages de vulgarisation, programmes de recherche-action, partenariat avec des acteurs locaux souvent plus sensibles à ces questions que les décideurs publics nationaux. En cela, ils sont tous trois des intellectuels spécifiques qui transforment leurs dispositions politiques en libido sciendi et, inversement, leurs travaux scientifiques nourrissent leur engagement public, Annie Thébaud-Mony étant certainement, sous ce rapport, la plus politique des trois. Leur point commun principal est d'appartenir à la petite communauté épistémique de chercheurs français qui s'efforcent, depuis plus de trente ans, de contourner le déni d'agenda des institutions, qu'elles soient scientifiques ou politiques, en matière d'ISS.

Finissons cependant sur une note positive : la montée des préoccupations environnementales en lien avec la santé, les critiques de plus en plus fortes sur le niveau des inégalités et leurs conséquences politiques (Piketty, 2019; Gethin et al., 2021), la forte augmentation de la prévalence des maladies chroniques, l'émergence dans le débat public de nouvelles catégories comme l' « universalisme proportionné » ou l' « investissement social » - et la critique de plus en plus fréquente de notions comme l' « égalité des chances », le « mérite » (Duru-Bellat, 2019) ou l'« équité » qui ont structuré le débat public depuis les années 1990 - pourraient, dans les prochaines années, conférer une plus grande audience à des travaux qui, pour le moment, demeurent cantonnés dans un secteur périphérique de l'expertise et de l'action publiques. 


\section{Références bibliographiques}

Bergeron H. et al. (2018), Le biais comportementaliste, Paris, Presses de Sciences Po.

Case A. et Deaton A. (2021), Morts de désespoir. L'avenir du capitalisme, Paris, Presses universitaires de France.

Castel R. (1995), Les métamorphoses de la question sociale, Paris, Fayard.

Cicolella A. (2013), Toxique planète. Le scandale invisible des maladies chroniques, Paris, Seuil.

Cobb Roger W. et Ross M. (1997), Cultural Strategies of Agenda Denial, Lawrence, University Press of Kansas.

Duru-Bellat M. (2019), Le mérite contre la justice, Paris, Presses de Sciences Po.

Fassin D. (2020), De l'inégalité des vies, Collège de France-Fayard.

Gethin A. et al. (dir.) (2021), Clivages politiques et inégalités sociales, Paris, Gallimard.

Haut Conseil de la santé publique (1994), La Santé en France, La Documentation française. Haut Conseil de la santé publique (2009), Inégalités sociales de santé : sortir de la fatalité, La Documentation française.

Henry E. (2017), Ignorance scientifique, inaction publique, Paris, Presses de Sciences Po.

Horton R. (2020), « Covid-19 is not a pandemic », The Lancet, 396 (26).

Institute of Health Equity (2020), Health Equity in England: The Marmot Review 10 Years On.

Jobert B. (dir.) (1994), Le tournant néolibéral en Europe, Paris, L’Harmattan.

Lang T. (2015), "Inégalités sociales de santé » In Henry E. et al., Dictionnaire critique de l'expertise, Paris, Presses de Sciences Po, p. 187-194.

Lang T. (2018), «Si nous considérions la réduction des inégalités sociales de santé comme l'affaire de tous ? », Santé publique, Vol. 30, Supplément 1.

Lang T. et al. (2020), « La santé des enfants en France : un enjeu négligé ?», Santé publique, 32(4), p. 329-338.

Leclerc A. et al. (dir.) (2000), Les inégalités sociales de santé, Paris, La Découverte.

Leclerc A. et al. (dir.) (2008), Inégaux face à la santé. Du constat à l'action, Paris, La Découverte.

Loretti A. (2021), La fabrique des inégalités sociales de santé, Rennes, Presses de l'EHESP.

Maurin L. (2021), Encore plus! Enquête sur ces privilégiés qui n'en ont jamais assez, Paris, Plon.

Noiriel G. (2010), Dire la vérité au pouvoir. Les intellectuels en question, Marseille, Agone.

Palier B. (2005), Gouverner la Sécurité sociale, Paris, Presses universitaires de France.

Pickett K. et Wilkinson R. (2013), Pourquoi l'égalité est meilleure pour tous, Paris, Les Petits matins.

Piketty T. (2019), Capital et idéologie, Paris, Seuil.

Pinell P. (2005), « Champ médical et processus de spécialisation », Actes de la recherche en sciences sociales, ${ }^{\circ} 156-157$, p. 4-36.

Rey O. (2020), L'idolatrie de la vie, Paris, Gallimard, collection « Tracts ».

Sandel Michael J. (2021), La tyrannie du mérite, Paris, Albin Michel.

Santi P. (2021), «La survie après un cancer s'améliore globalement, malgré de fortes disparités ", Le Monde, 6 juillet.

Stiegler B. (2021), De la démocratie en pandémie, Paris, Gallimard, collection « Tracts ».

Thébaud-Mony A. (2021), Politiques assassines et luttes pour la santé au travail, Paris, La Dispute. 